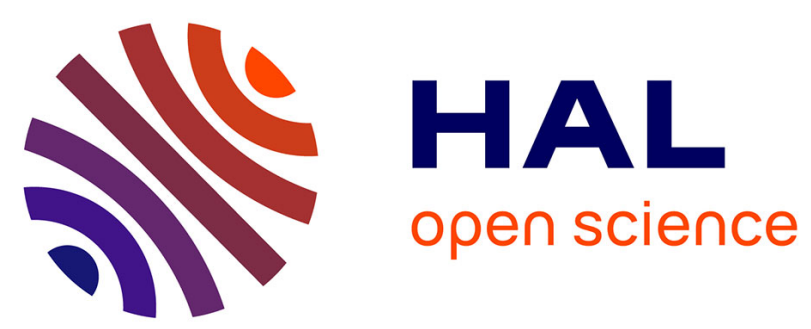

\title{
Recurrence quantification analysis of surface electromyogram supports alterations in motor unit recruitment strategies by anodal transcranial direct current stimulation
}

\author{
Anirban Dutta, Chandramouli Krishnan, Shailesh S. Kantak, Rajivc
}

Ranganathan, Michael A. Nitsche

\section{To cite this version:}

Anirban Dutta, Chandramouli Krishnan, Shailesh S. Kantak, Rajivc Ranganathan, Michael A. Nitsche. Recurrence quantification analysis of surface electromyogram supports alterations in motor unit recruitment strategies by anodal transcranial direct current stimulation. Restorative Neurology and Neuroscience, 2015, 33 (5), pp.663-669. 10.3233/RNN-140469 . hal-01203368

\section{HAL Id: hal-01203368 \\ https://hal.inria.fr/hal-01203368}

Submitted on 17 May 2019

HAL is a multi-disciplinary open access archive for the deposit and dissemination of scientific research documents, whether they are published or not. The documents may come from teaching and research institutions in France or abroad, or from public or private research centers.
L'archive ouverte pluridisciplinaire HAL, est destinée au dépôt et à la diffusion de documents scientifiques de niveau recherche, publiés ou non, émanant des établissements d'enseignement et de recherche français ou étrangers, des laboratoires publics ou privés. 


\title{
Recurrence quantification analysis of surface electromyogram supports alterations in motor unit recruitment strategies by anodal transcranial direct current stimulation
}

\author{
Anirban Dutta $^{\mathrm{a}^{*}}$, Chandramouli Krishnan ${ }^{\mathrm{b}, \mathrm{c} \varpi *}$, Shailesh S. Kantak ${ }^{\mathrm{c}, \mathrm{d}}$, Rajiv Ranganathan ${ }^{\mathrm{c}, \mathrm{e}}$, Michael A. \\ Nitsche ${ }^{\mathrm{f}}$ \\ *indicates equal contribution \\ ${ }^{a}$ DEMAR team of INRIA, Université de Montpellier, CNRS,34095 Montpellier Cedex 5, France \\ ${ }^{\mathrm{b}}$ Neuromuscular and Rehabilitation Robotics Laboratory (NeuRRO Lab), Department of Physical \\ Medicine and Rehabilitation,University of Michigan,Ann Arbor, MI, USA \\ ${ }^{\mathrm{c}}$ Department of Physical Medicine and Rehabilitation, Northwestern University Feinberg School of \\ Medicine, Chicago, IL, USA. \\ ${ }^{\mathrm{d}}$ Moss Rehabilitation Research Institute, Elkins Park, PA, USA. \\ ${ }^{\mathrm{e}}$ Department of Kinesiology, Michigan State University, East Lansing, MI, USA. \\ ${ }^{\mathrm{d}}$ Department of Clinical Neurophysiology, University Medical Center, 37075 Göttingen, Germany
}

Corresponding Author:

Dr. Chandramouli Krishnan

Department of Physical Medicine \& Rehabilitation

University of Michigan

325 E Eisenhower Parkway, Suite 3013

Ann Arbor, MI - 48108

Phone: +1-319-321-0117

Email: mouli@umich.edu 


\section{$\underline{\text { ABSTRACT }}$}

Purpose: Recent evidence indicates that anodal transcranial direct current stimulation (tDCS) can selectively alter the EMG/force relationship of agonist arm muscles; however, the mechanisms mediating those changes are less clear. The purpose of this study was to evaluate the effect of anodal tDCS on motor unit synchronization by using a sophisticated non-linear EMG analysis called as recurrence quantification analysis (RQA). Methods: Surface EMG signals were collected from the biceps brachii muscle of eighteen healthy young adults (9 tDCS and 9 control) at various force levels (12.5\%, $25 \%, 37.5 \%$, and $50 \%$ maximum) before and after the application of anodal tDCS over the primary motor cortex. RQA was employed to quantify the changes in percentage of determinism (\%DET) and laminarity (\%LAM) of the surface EMG signals, which are surrogate measures of motor unit synchronization. Results: RQA analyses indicated that the changes in \%DET and \%LAM scores were significantly higher in the tDCS group than in the control group $(\mathrm{p}<0.05)$ and this effect was particularly pronounced at higher force levels. Conclusion: The results of this study provide novel evidence supporting that anodal tDCS significantly alters motor unit firing strategies (i.e., the degree of synchronization) of the biceps brachii muscle.

Keywords: Recurrence analysis; electromyography; nonlinear analysis; brain stimulation; motor unit; synchronization 


\section{$\underline{\text { INTRODUCTION }}$}

A growing body of evidence indicates that cortical neural activity can be noninvasively modulated by the application of transcranial direct current stimulation (tDCS) (Nitsche and Paulus, 2000, 2011; Schade et al., 2012). Recent work by Krishnan and colleagues also indicates that anodal tDCS can selectively affect the voluntary electromyogram $(\mathrm{EMG})$ /force relationship of agonist muscles without altering the coactivation of antagonistic muscles (Krishnan et al., 2014). The authors theorized that the observed greater EMG activity per unit force after anodal tDCS was related to alterations in motor unit recruitment or firing strategies (e.g., motor unit synchronization). They, however, did not perform formal EMG analysis to support their hypothesis. Recurrence quantification analysis (RQA) is a sensitive nonlinear analysis tool for studying synchronization in human motor systems (Del Santo et al., 2007; Del Santo et al., 2006; Farina et al., 2002). In RQA, subtle changes in motor unit synchronization can be noninvasively quantified using the percentage of determinism (\%DET), which reflects the amount of rule-obeying structure in the signal dynamics (Webber et al., 1995) and reveals embedded determinisms in apparently stochastic signals, such as surface EMG. Indeed, Del Santo and colleagues (Del Santo et al., 2007) showed that the \%DET obtained from RQA is as effective as cross-correlation analysis of single motor unit activity, which is the conventional gold standard technique for studying motor unit synchronization. Thus, the current study evaluated the alterations in motor unit synchronization due to anodal tDCS by studying the changes in \%DET values obtained from RQA (Filligoi and Felici, 1999).

\section{MATERIALS \& METHODS}

\section{A. Subjects}

Eighteen young adults ( 9 experimental and 9 control subjects; Age: $28.4 \pm 4.8$ years; Height: $1.72 \pm$ 0.08 meters; Weight: $67.9 \pm 10.0$ kilograms) participated in this study. Subjects were part of another 
study that has been published elsewhere (Krishnan et al., 2014). All subjects were screened for the presence of neurological or orthopedic impairments and for any contraindications to transcranial magnetic stimulation (TMS). Subjects in both groups were age- and gender-matched to minimize the confounding effects of these factors on EMG/force relationship. Before enrollment, a brief description of the study was provided to all subjects and written informed consent approved by the Northwestern University Institutional Review Board was obtained.

\section{B. Experimental protocol}

The experimental protocol involved collecting of surface EMG signals (sampling rate: $2000 \mathrm{~Hz}$, filter cut-off: 20 to $500 \mathrm{~Hz}$ ) from the subject's dominant arm before and after the application of anodal tDCS over the primary motor cortex (see Figure 1a). After preparing the skin over the electrode site, disposable surface EMG electrodes (inter-electrode distance $=1.75 \mathrm{~cm}$ ) were placed over the muscle belly of the biceps brachii muscle. A common reference electrode was placed on the skin over the lateral epicondyle of the humerus. The subject then stood on a flat platform with their palm placed over the force sensing arm of a custom designed load cell unit. After performing maximum voluntary isometric contractions (MVICs), the subject matched linear force targets $(12.5 \%, 25 \%, 37.5 \%$, and $50 \%$ of maximum) projected on a computer monitor by exerting elbow flexor force over the load sensing arm for five seconds. All subjects performed three trials at each force level and the order in which the force targets appeared was randomized. A 45-second rest period was provided between each trial to minimize any potential effects of fatigue. After completing the target-matching experiment, the subject underwent either anodal tDCS (2 mA, 10 minutes, $0.125 \mathrm{~mA} / \mathrm{cm}^{2}$ ) (Laczo et al., 2014; Wiethoff et al., 2014) or the control condition depending on their group allocation. All procedures were exactly identical between the two conditions (i.e., groups), except that no stimulation was provided to the subjects in the control condition (see Figure 1a). Anodal tDCS was applied using a battery operated constant current stimulator (Dupel Iontophoresis System, EMPI, MN, USA). The saline $(0.09 \% \mathrm{NaCl})$ soaked anode $\left(16 \mathrm{~cm}^{2}\right)$ was 
placed over the hot spot of the biceps brachii muscle identified by transcranial magnetic stimulation (TMS), and the return electrode $\left(54 \mathrm{~cm}^{2}\right)$ was placed over the contralateral supraorbital region. All subjects tolerated tDCS well with mild tingling and itching sensation being the only complaints. Following the completion of tDCS application, the target-matching experiment was repeated in an identical fashion to obtain post-tDCS EMG activity of the biceps brachii muscle.

\section{Recurrence quantification analysis}

RQA is able to detect the deterministic structure of the underlying dynamical process of a time series which is based on the time delay embedding procedure for phase space reconstruction (Marwan et al., 2013). EMG data recorded from the Biceps brachii (agonist) muscle during each trial across a range of force levels (12.5\%-50\% of maximum) was used for RQA. RQA was conducted offline from 2.56s of the central period of the EMG signal (5120 samples). For each epoch, \%DET and \%Laminarity (\%LAM), i.e., analogous to \%DET except that it measures the percentage of recurrent points comprising vertical line structures rather than diagonal line structures (Marwan et al., 2002), were computed. The first step in RQA was an embedding procedure, where vectors representing the states of the system were extracted (Marwan et al., 2013). To determine the embedding dimension, the false nearest neighbors method (Kennel et al., 1992) was used which was set to 15 . Therefore, the phase space was assumed to be 15-dimensional, in accordance with parameters chosen in other studies (Dutta et al., 2012; Farina et al., 2002; Webber et al., 1995). Then, 15 dimensional vectors were extracted from 2.56s epoch of the surface EMG signal by lagging the signal by an integer number of samples that minimized the temporal correlations between the samples of the time series. This integer number was given by the first local minimum of the average mutual information function (Fraser and Swinney, 1986). This lag was set at 6 since most epochs gave us that integer number. Then, the recurrence plot was constructed to easily get insight into the high-dimensional dynamical systems (Eckmann et al., 1987). For constructing the recurrent plot, we used the Cross Recurrence Plot Toolbox - CRPtoolbox 3.20.9 (Marwan et al., 2007). 
From the recurrence plot, we extracted \%DET and \%LAM using CRPtoolbox ('crqa' function) for a minimum diagonal line $=3$ and minimum vertical line $=3$. We used the shuffled surrogates test (Marwan et al., 2007) to test the null hypothesis that the data are produced by a purely random uncorrelated process.

\section{Data Analyses}

Statistical analyses were performed using SPSS v.22 for Windows (SPSS Inc., Chicago, IL, USA). $\% \mathrm{DET}$ and \%LAM variables obtained from the RQA were normalized by computing a change score (equation 1) and averaged across the three trials.

$$
\begin{aligned}
\% D E \text { Tnorm } & =\frac{\% D E T_{\text {post }}-\% D E T_{\text {pre }}}{\% D E T_{\text {pre }}} \\
\% L A M \text { norm } & =\frac{\% L A M_{\text {post }}-\% L A M_{\text {pre }}}{\% L A M_{\text {pre }}}
\end{aligned}
$$

where the subscript pre and post denotes the pre- and post- tDCS intervention values. Repeated measures analysis of variance (ANOVA) with MVIC level (12.5\%, 25\%, 37.5\%, 50\%) as within-subject factor and condition (tDCS, control) as a between-subjects factor was performed to detect the effect of anodal tDCS on \%DETnorm and \%LAMnorm. In case of significant main or interaction effects, posthoc analyses using t-tests were conducted. A significance level of $\alpha=0.05$ was used for all analyses.

\section{RESULTS}

The actual force exerted by subjects during submaximal isometric target-matching was similar to the target force in all conditions (tDCS and control) and sessions (pre and post), indicating that the accuracy with which subjects performed the task was very good and did not confound the results (see Figure 1b). The shuffled surrogates resulted in a systematic and dramatic drop of $\%$ DET and \%LAM to $\sim 0 \%$ which showed that random shuffling destroyed all temporal structures revealed by the \%DET and \%LAM parameters of the recorded signals. Figure 2a shows a typical recurrence plot of an EMG epoch, which 
displays also the respective \%DET and \%LAM. The repeated measures ANOVAs revealed a significant main effect of condition for both \%DETnorm $(\mathrm{F}(1,16)=7.560, \mathrm{p}=0.014)$ (see Figure $2 \mathrm{~b}$ ) and \%LAMnorm $(\mathrm{F}(1,16)=8.225, \mathrm{p}=0.011)$ (see Figure $2 \mathrm{~b}$ ), but no significant effect of MVIC level for \%DETnorm $(\mathrm{F}(3,48)=0.312, \mathrm{p}=0.817)($ see Figure $2 \mathrm{c})$ or \%LAMnorm $(\mathrm{F}(3,48)=0.336, \mathrm{p}=0.799)($ see Figure 2d). There was also no significant interaction effect between condition and MVC level for \%DETnorm $(\mathrm{F}(3,48)=0.392, \mathrm{p}=0.759)($ see Figure $2 \mathrm{c})$ or \%LAMnorm $(\mathrm{F}(3,48)=0.351, \mathrm{p}=0.789)(\mathrm{see}$ Figure 2d). Post-hoc analyses revealed that the \%DETnorm and \%LAMnorm values were significantly higher in the tDCS group as compared to the control group and this effect was particularly pronounced at higher MVIC levels ( $p=0.015$ and $p=0.012$, see Figure 3).

\section{$\underline{\text { DISCUSSION }}$}

The novel finding of this work is that \%DET and \%LAM are influenced by the application of anodal tDCS to the primary motor cortex. Specifically, anodal tDCS increased the \%DET and \%LAM of the Biceps brachii surface EMG signals. Here, \%LAM is analogous to \%DET except that it measures the percentage of recurrent points comprising vertical line structures rather than diagonal line structures (Marwan et al., 2002). Since \%DET has been shown to depend on motor unit synchronization (Farina et al., 2002) (i.e., a physiological condition where two motor units fire simultaneously or nearsimultaneously), the post-intervention increase in \%DET (and \%LAM) following anodal tDCS is most likely related to an increased level of motor unit synchronization.

According to the common physical-connection mechanism theory (De Luca et al., 1993), synchronized firing of two motor units occur due to the existence of common physical connections between them (e.g., through branches of common stem presynaptic fibers from peripheral, segmental, or supraspinal sources) (Farmer et al., 1993; Schmied et al., 1999). Therefore, it is possible that anodal tDCS could have increased the strength of common corticospinal drive to spinal motor neurons, which would then have increased the extent of motor unit synchronization (Semmler, 2002). Alternatively, 
alterations to additional common inputs from secondary sources (e.g., Ia afferents) via segmental or supraspinal mechanisms could have increased the extent of motor unit synchronization (Defreitas et al., 2014; Lackmy-Vallee et al., 2014; Maltenfort et al., 1998; Roche et al., 2009, 2011). Further research incorporating corticomuscular (EEG-EMG) correlation/coherence analysis and-or H-reflex analysis may elucidate the potential sources of tDCS mediated motor unit synchronization.

It is important to note that the changes in $\% \mathrm{DET}$ and \%LAM values were particularly greater at higher MVIC levels. While the precise reason for this observation is not clear, it appears that motor unit synchronization is typically greater at higher force levels than at lower force levels (Fling et al., 2009). Further, high-threshold motor units, which are typically recruited at higher force levels, are known to exhibit greater synchronization than low-threshold motor units due to differences in strength of synaptic inputs from spindle afferents (which are known to have a desynchronizing effect) (Defreitas et al., 2014). However, a limitation of the current study was that the muscle activation patterns at force levels beyond $50 \%$ MVIC was not evaluated, as we wanted to minimize the confounding effects of fatigue and metaplasticity (Muller-Dahlhaus and Ziemann, 2014; Thirugnanasambandam et al., 2011). As a result, it is not clear whether greater changes would have been observed at higher force levels (i.e., beyond 50\% of maximum), particularly considering that biceps brachii exhibits a wide recruitment range $(0-88 \%$ of MVIC) (Kukulka and Clamann, 1981). Because motor unit synchronization is known to affect the amplitude of the surface EMG signals (Yao et al., 2000), it is likely that the observed tDCS-mediated increase in EMG activation by Krishnan and colleagues (Krishnan et al., 2014) can be at least be partly attributed to higher motor unit synchronization, thereby supporting the ability of anodal tDCS to alter motor unit recruitment strategies.

\section{$\underline{\text { REFERENCES }}$}

De Luca, C.J., Roy, A.M. and Erim, Z. (1993). Synchronization of motor-unit firings in several human muscles. J Neurophysiol, 70(5), 2010-2023. 
Defreitas, J.M., Beck, T.W., Ye, X. and Stock, M.S. (2014). Synchronization of low- and high-threshold motor units. Muscle Nerve, 49(4), 575-583.

Del Santo, F., Gelli, F., Mazzocchio, R. and Rossi, A. (2007). Recurrence quantification analysis of surface EMG detects changes in motor unit synchronization induced by recurrent inhibition. Exp Brain Res, 178(3), 308-315.

Del Santo, F., Gelli, F., Schmied, A., Vedel, J.P., Rossi, A. and Mazzocchio, R. (2006). Motor unit synchronous firing as revealed by determinism of surface myoelectric signal. J Neurosci Methods, 155(1), 116-121.

Dutta, A., Khattar, B. and Banerjee, A. (2012). Nonlinear analysis of electromyogram following gait training with myoelectrically triggered neuromuscular electrical stimulation in stroke survivors. Eurasip J Adv Signal Process 1531-8.

Eckmann, J.P., Kamphorst, S.O. and Ruelle, D. (1987). Recurrence plots of dynamical systems. Europhys Lett 4(9), 973.

Farina, D., Fattorini, L., Felici, F. and Filligoi, G. (2002). Nonlinear surface EMG analysis to detect changes of motor unit conduction velocity and synchronization. $J$ Appl Physiol (1985), 93(5), 1753 1763.

Farmer, S.F., Bremner, F.D., Halliday, D.M., Rosenberg, J.R. and Stephens, J.A. (1993). The frequency content of common synaptic inputs to motoneurones studied during voluntary isometric contraction in man. J Physiol, 470127-155.

Filligoi, G. and Felici, F. (1999). Detection of hidden rhythms in surface EMG signals with a non-linear time-series tool. Med Eng Phys, 21(6-7), 439-448.

Fling, B.W., Christie, A. and Kamen, G. (2009). Motor unit synchronization in FDI and biceps brachii muscles of strength-trained males. J Electromyogr Kinesiol, 19(5), 800-809.

Fraser, A.M. and Swinney, H.L. (1986). Independent coordinates for strange attractors from mutual information. Phys Rev A, 33(2), 1134-1140.

Kennel, M.B., Brown, R. and Abarbanel, H.D. (1992). Determining embedding dimension for phasespace reconstruction using a geometrical construction. Phys Rev A, 45(6), 3403-3411.

Krishnan, C., Ranganathan, R., Kantak, S.S., Dhaher, Y.Y. and Rymer, W.Z. (2014). Anodal transcranial direct current stimulation alters elbow flexor muscle recruitment strategies. Brain Stimul, 7(3), 443-450.

Kukulka, C.G. and Clamann, H.P. (1981). Comparison of the recruitment and discharge properties of motor units in human brachial biceps and adductor pollicis during isometric contractions. Brain Res, 219(1), 45-55.

Lackmy-Vallee, A., Klomjai, W., Bussel, B., Katz, R. and Roche, N. (2014). Anodal transcranial direct current stimulation of the motor cortex induces opposite modulation of reciprocal inhibition in wrist extensor and flexor. $J$ Neurophysiol, 112(6), 1505-1515. 
Laczo, B., Antal, A., Rothkegel, H. and Paulus, W. (2014). Increasing human leg motor cortex excitability by transcranial high frequency random noise stimulation. Restor Neurol Neurosci, 32(3), 403-410.

Maltenfort, M.G., Heckman, C.J. and Rymer, W.Z. (1998). Decorrelating actions of Renshaw interneurons on the firing of spinal motoneurons within a motor nucleus: a simulation study. $J$ Neurophysiol, 80(1), 309-323.

Marwan, N., Carmen Romano, M., Thiel, M. and Kurths, J. (2007). Recurrence plots for the analysis of complex systems. Phys Rep, 438(5), 237-329.

Marwan, N., Schinkel, S. and Kurths, J. (2013). Recurrence plots 25 years later-Gaining confidence in dynamical transitions. Europhys Lett, 101(2 ), 20007.

Marwan, N., Wessel, N., Meyerfeldt, U., Schirdewan, A. and Kurths, J. (2002). Recurrence-plot-based measures of complexity and their application to heart-rate-variability data. Phys Rev E Stat Nonlin Soft Matter Phys, 66(2 Pt 2), 026702.

Muller-Dahlhaus, F. and Ziemann, U. (2014). Metaplasticity in Human Cortex. Neuroscientist.

Nitsche, M.A. and Paulus, W. (2000). Excitability changes induced in the human motor cortex by weak transcranial direct current stimulation. $J$ Physiol, 527 Pt 3633-639.

Nitsche, M.A. and Paulus, W. (2011). Transcranial direct current stimulation--update 2011. Restor Neurol Neurosci, 29(6), 463-492.

Roche, N., Lackmy, A., Achache, V., Bussel, B. and Katz, R. (2009). Impact of transcranial direct current stimulation on spinal network excitability in humans. J Physiol, 587(Pt 23), 5653-5664.

Roche, N., Lackmy, A., Achache, V., Bussel, B. and Katz, R. (2011). Effects of anodal transcranial direct current stimulation over the leg motor area on lumbar spinal network excitability in healthy subjects. $J$ Physiol, 589(Pt 11), 2813-2826.

Schade, S., Moliadze, V., Paulus, W. and Antal, A. (2012). Modulating neuronal excitability in the motor cortex with tDCS shows moderate hemispheric asymmetry due to subjects' handedness: a pilot study. Restor Neurol Neurosci, 30(3), 191-198.

Schmied, A., Pouget, J. and Vedel, J.P. (1999). Electromechanical coupling and synchronous firing of single wrist extensor motor units in sporadic amyotrophic lateral sclerosis. Clin Neurophysiol, 110(5), 960-974.

Semmler, J.G. (2002). Motor unit synchronization and neuromuscular performance. Exerc Sport Sci Rev, $30(1), 8-14$.

Thirugnanasambandam, N., Sparing, R., Dafotakis, M., Meister, I.G., Paulus, W., Nitsche, M.A. and Fink, G.R. (2011). Isometric contraction interferes with transcranial direct current stimulation (tDCS) induced plasticity: evidence of state-dependent neuromodulation in human motor cortex. Restor Neurol Neurosci, 29(5), 311-320. 
Webber, C.L., Jr., Schmidt, M.A. and Walsh, J.M. (1995). Influence of isometric loading on biceps EMG dynamics as assessed by linear and nonlinear tools. J Appl Physiol (1985), 78(3), 814-822.

Wiethoff, S., Hamada, M. and Rothwell, J.C. (2014). Variability in response to transcranial direct current stimulation of the motor cortex. Brain Stimul, 7(3), 468-475.

Yao, W., Fuglevand, R.J. and Enoka, R.M. (2000). Motor-unit synchronization increases EMG amplitude and decreases force steadiness of simulated contractions. $J$ Neurophysiol, 83(1), 441-452.

\section{FiGURE LEGEND}

Figure 1: A) Schematic of the experimental design. B) Line plots demonstrating the actual vs. target force. Note that the actual force was similar to the target force in all conditions (tDCS and control) and sessions (pre and post), indicating that the accuracy with which subjects performed the task was very good.

Figure 2: A) An illustrative recurrence map and its percentage of determinism (\%DET) and Laminarity (\%LAM). Note that the recurrence quantification analysis parameters were, embedding dimension=15, embedding delay $=6$, and minimum diagonal line $=3$, minimum vertical line $=3$. B) Post $-h o c$ tests indicating the population marginal means of \%DETnorm (top panel) and \%LAMnorm (bottom panel) for the tDCS and control condition. C) Analysis of variance (ANOVA) for \%DETnorm, D) ANOVA for \%LAMnorm.

Figure 3: Scatterplots demonstrating the effect of anodal tDCS on normalized changes in (A) percentage of determinism (\%DETnorm) and (B) laminarity (\%LAMnorm) of the surface EMG signals at various force levels $(12.5 \%, 25 \%, 37.5 \%$, and $50 \%$ of maximum). Error bars indicate standard error of the mean (SEM) and solid lines indicate best fit regression lines. 


\section{Figure 1}

(A)

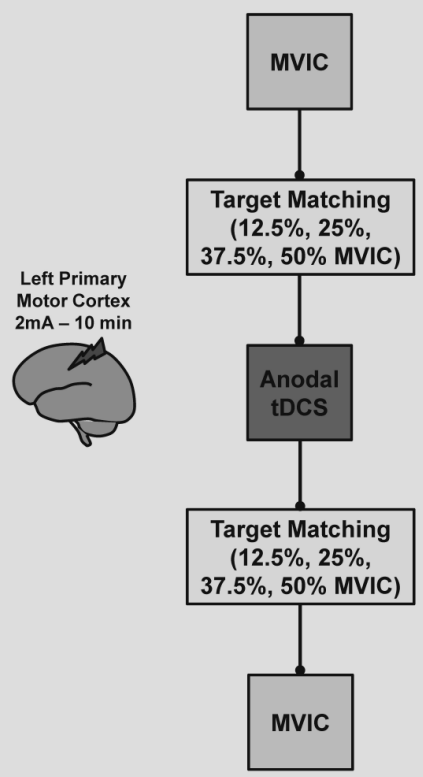

(B)

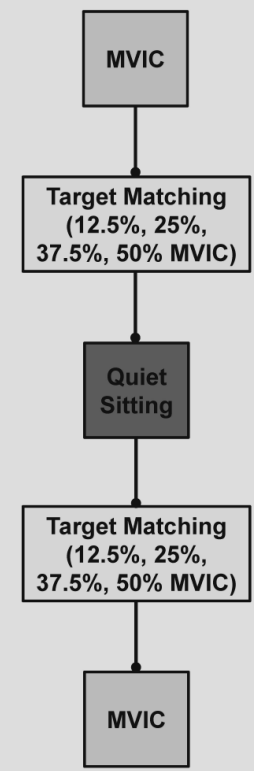

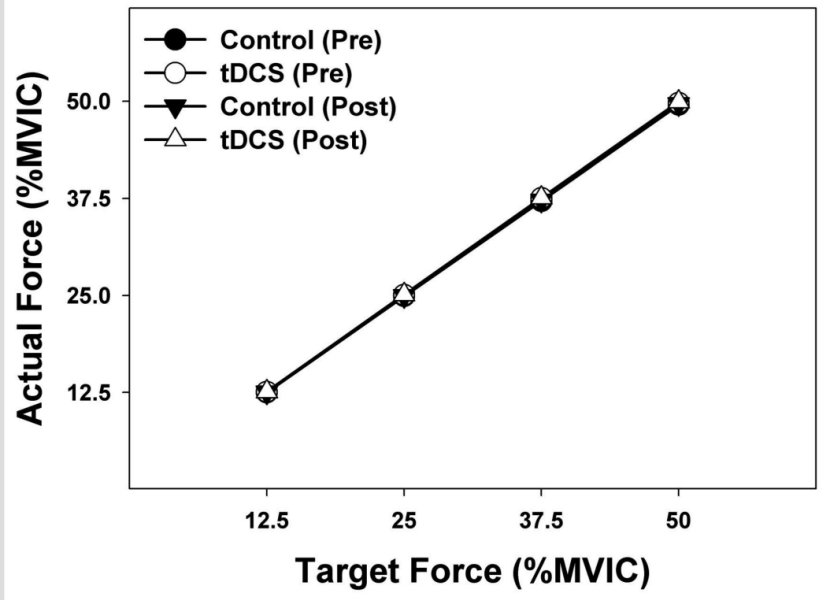




\section{Figure 2}

(A)

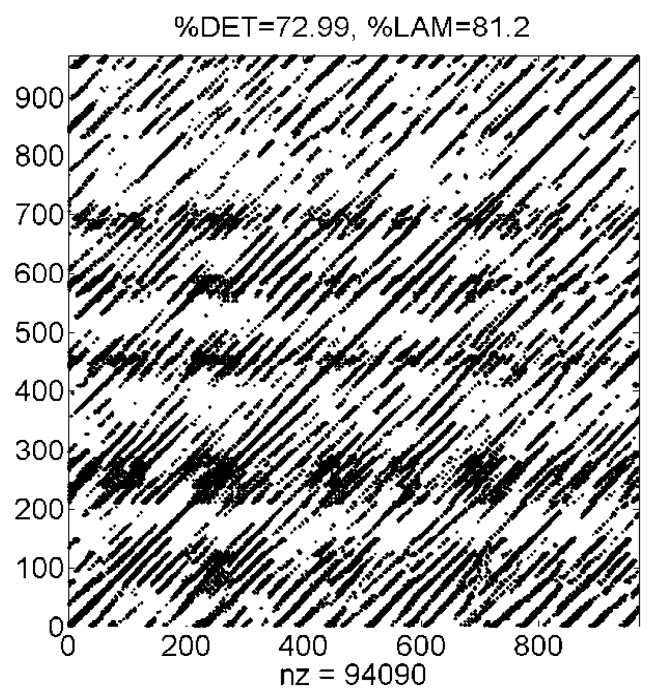

(B)

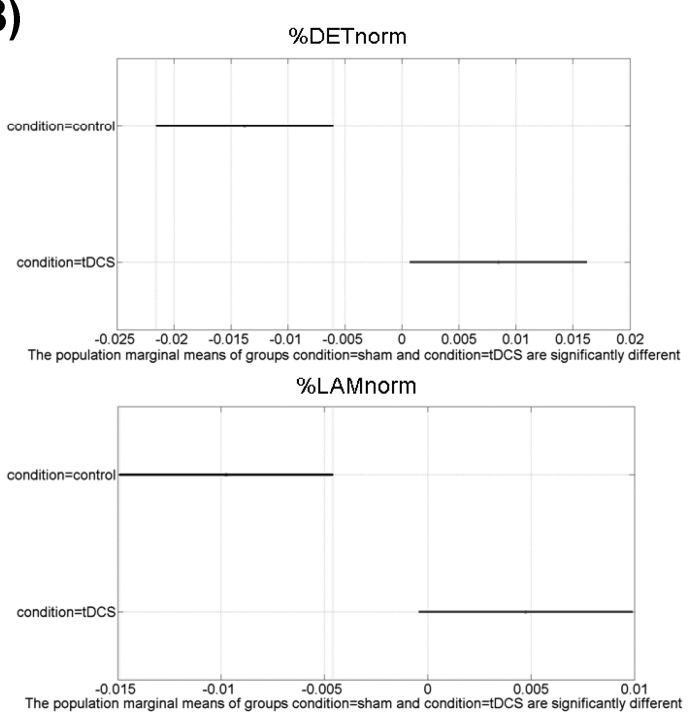

(C)

(D)

\begin{tabular}{lccccc}
\multicolumn{9}{c}{ Analysis of Variance } & & \\
\hline Source & Sum Sq. & d.f. & Mean Sq. & F & Significance \\
\hline Condition & 0.009 & 1 & 0.009 & 7.560 & $0.014^{*}$ \\
MVIC Level & 0.001 & 3 & 0.000 & 0.312 & 0.817 \\
Condition*MVIC Level & 0.001 & 3 & 0.000 & 0.392 & 0.759 \\
Error (Condition) & 0.019 & 16 & 0.001 & & \\
Error (MVIC Level) & 0.057 & 48 & 0.001 & & \\
\hline
\end{tabular}




\section{Figure 3}

(A)

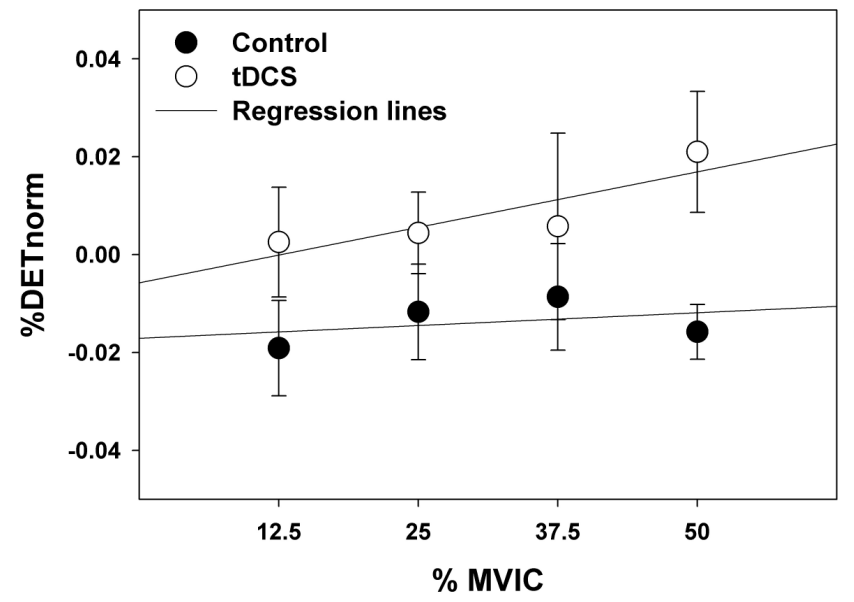

(B)

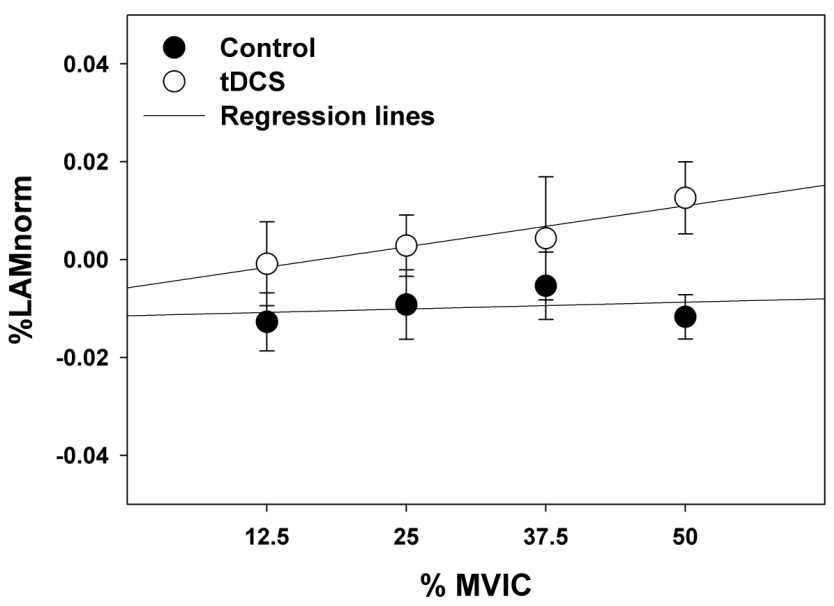

